\title{
Do goal cue and motor activity impact preschoolers' working memory?
}

\author{
Christophe Fitamen*1,2, Agnès Blaye ${ }^{2}$ and Valérie Camos ${ }^{1}$ \\ 'Département de Psychologie, Université de Fribourg, Switzerland \\ ${ }^{2}$ Laboratoire de Psychologie Cognitive, Université d'Aix-Marseille \& CNRS, France
}

\begin{abstract}
Preschoolers are well known for their poor working memory (WM) performance. This could result from goal neglect, which would hamper the setting of maintenance strategies. Previous studies have shown that preschoolers' WM performance can be improved in game-like tasks, because they provide cues to support goal maintenance. However, in these studies, it was unclear what features of the task (either the main toy or the motor activity required by the game) provide efficient cues. The aim of the present study was to disentangle the two features to examine cue effects in 5- to 7-year-old children. No improvement of WM performance was observed when the toy was a potential goal cue, whereas the motor activity had a detrimental effect in all age groups. The latter effect could result from a distraction of attention from attention-based maintenance activities. Hence, preschoolers' poor WM performance would not be fundamentally due to goal neglect.
\end{abstract}

\section{Statement of contribution}

\section{What is already known on this subject?}

- Goal cues have proved to improve preschoolers' performance in executive control.

- Motor activity can improve preschoolers' working memory performance if it is a meaningful activity.

- Motor activity can also decrease children's working memory if resources need to be shared.

\section{What does this study add?}

- Cueing does not improve 5- to 7-year-olds' working memory performance.

- Walking to the goal is detrimental to working memory performance in all age groups.

- Impaired 5-years-olds' working memory by walking can be the mark of an early implementation of an attentional maintenance mechanism.

\section{Background}

Working memory (WM) has been studied for more than four decades, and many conceptions coexist. However, a generic and consensual definition would be that 'working memory refers to the ensemble of components of the mind that hold a limited amount of information temporarily in a heightened state of availability for use in ongoing information processing' (Cowan, 2016, 14-15). Because of this central role in human

* Correspondence should be addressed to Christophe Fitamen, Département de Psychologie, Université de Fribourg, Rue de Faucigny 2, CH- I 700 Fribourg, Switzerland (email: christophe.fitamen@unifr.ch). 
cognition, WM development is considered as a major source of cognitive development, and WM capacity is the best predictor of achievement in cognitive tasks and school achievements (see Camos \& Barrouillet, 2018; Gathercole, Lamont, \& Alloway, 2006, for review; Swanson, 1999).

Across childhood, WM capacity increases steadily, although two main developmental periods can be roughly distinguished. Before the age of 6-7 years, children have difficulties counteracting the loss of memory traces, which suggests that they either have no information-maintenance mechanisms available or cannot implement them adequately (although many questions are still unsolved; see Camos \& Barrouillet, 2011). By contrast, children after the age of 6-7 are able to use various maintenance mechanisms to counteract memory loss (e.g., Allik \& Siegel, 1976; Barrouillet, Gavens, Vergauwe, Gaillard, \& Camos, 2009; Camos \& Barrouillet, 2011; Flavell, Beach, \& Chinsky, 1966; Hitch \& Halliday, 1983; Oftinger \& Camos, 2016, 2017, 2018; Tam, Jarrold, Baddeley, \& Sabatos-DeVito, 2010). To account for the developmental change observed between the two age periods (i.e., before and after 6-7), it was recently proposed that preschoolers may neglect the 'memorize' goal of the WM tasks (Fitamen, Blaye, \& Camos, 2019). This goal neglect could hence impair the triggering of the maintenance strategies and lead to the poor memory performance reported in preschoolers and the difficulties to counteract memory loss. Indeed, it is now well known that preschoolers have difficulty keeping the goal active during executive control tasks (Chevalier \& Blaye, 2008; Marcovitch, Boseovski, \& Knapp, 2007; Marcovitch, Boseovski, Knapp, \& Kane, 2010; Yanaoka \& Saito, 2017). It is thus reasonable to think that it could also be at stake in WM tasks that are executive tasks in that they often request to switch between the maintenance of information and a secondary task.

In executive control tasks, cues provided to favour the maintenance of the goal have been shown to reduce goal neglect. Exogenous cues are the type of cues mostly studied in the field of executive functions. By presenting an image or hearing a word referring to the goal of the task to perform in the upcoming trial of a task-switch paradigm, children improve their executive functioning (e.g., Chevalier \& Blaye, 2008). Moreover, the quality of the cueing influences the goal maintenance. For instance, transparent cues for which the relation to the goal is obvious are more effective in improving children's performance than arbitrary ones (Blaye \& Chevalier, 2011; Chevalier \& Blaye, 2009; Towse, Lewis, \& Knowles, 2007). The format of the exogenous cues also has an impact on their efficiency. An auditory-verbal cue is better at helping children maintaining the goal than a visual cue (Chevalier \& Blaye, 2009), bearing support to the hypothesis that goal is maintained in a verbal code (Gruber \& Goschke, 2004). Unlike the auditory-verbal cue, a visual cue needs to be transcoded to be used. Varying the nature (verbal vs. visual) of a cue presented during the delay of retention in WM task, Fitamen et al. (2019) showed that a visual cue (i.e., the drawing of an asexual character pointing a finger to her head in a thinking attitude) increased the use of articulatory rehearsal while a verbal-auditory cue (i.e., hearing the message 'remember the images') impaired WM performance. This latter finding was interpreted as resulting from the interference between the verbal items to memorize and the cue. Thus, and contrary to what is reported in executive control tasks, the use of visual cues should be favoured in verbal WM tasks.

It seems likely that aside from exogenous goal cues, some endogenous cues can favour the maintenance of goal. Considering school-aged children, they generally manage to engage information-maintenance mechanisms even in the absence of any exogenous cues. This suggests that they are able to rely on endogenous cues to keep the memorize goal active enough to trigger these strategies and/or that they are better able to resist to 
distractors that interfere with memorization during the delay between items encoding and recall time. As a consequence, when children are engaged in an activity, this creates a lasting and meaningful connection between encoding and recall times, which may constitute an endogenous cue that scaffolds the memorize goal maintenance and hence lead to improved memory performance. The developmental literature offers several examples of the cognitive benefit of different forms of motor activities. Goldin-Meadow and colleagues have shown that producing hand gestures during a problem-solving task can improve cognitive performance to the extent that it makes sense within the task in which gestures are performed (Cook \& Goldin-Meadow, 2006; Goldin-Meadow, Nusbaum, Kelly, \& Wagner, 2001). Furthermore, two independent studies on preschoolers' WM have suggested that a meaningful motor activity such as walking may improve children's performance (Bertrand \& Camos, 2015; Istomina, 1975).

In an original paradigm developed by Istomina (1975), memory performance of 3- to 7year-old children was compared in two situations, one rather static and one in which children had to move. More specifically, children were either involved in a situation similar to a laboratory test condition or played a shopping game. In the former situation akin to a lesson, a list of items was presented and children had to recall it. In the latter, the list of items was presented as a shopping list and children had to go to a toy shop and ask for the items to another child playing the merchant. Preschoolers exhibited better recall performance in the play than in the laboratory condition. Istomina concluded that the shopping game benefits memory performance by supporting goals of remembering and recalling (Istomina, 1975). Unfortunately, the contrast between the play and laboratory conditions included many confounds. When analysed in terms of cueing, the game-like situation implemented by Istomina (1975) was rich in exogenous cues due to the presence of a store, the items in the store, the other children, and the experimenter playing the employees of the store, but also rich in endogenous goal cues. Indeed, because walking is itself goal-directed and, in contrast with the instructions given at the outset of the task, lasts during the whole retention delay, it could well play the role of an endogenous goal cue, that would support goal maintenance in younger children. Following Istomina $(1975,60)$, 'the relationship between the goal and the act [playing] becomes itself an intrinsic relationship', as if each child would embody the goal to achieve.

Recently, Bertrand and Camos (2015) implemented a rather similar game situation to assess preschoolers' WM. The task was also a shopping game in which 4- to 6-year-old children had to memorize lists of verbal items for further recall either immediately after the presentation of the items or after a delay. They showed that WM performance improved when children had to walk straight to a toy shop instead of waiting seated in the front of the shop during the same delay. Among their interpretations, the authors suggested that walking being a goal-oriented motor activity, it may have improved goal maintenance in preschoolers and led to the observed better recall performance. However, in this study, the impact of the endogenous and exogenous cues cannot be disentangled. To summarize, both Istomina (1975) and Bertrand and Camos (2015) findings are consistent in suggesting that an enactment of a situation can help improving preschoolers' memory, but for both studies, the respective role of endogenous and exogenous cues cannot be examined independently.

The present study examined the effect of an exogenous goal cue and a motor activity in supporting the maintenance of goal in a WM task. Whereas both factors may have affected goal maintenance in previous studies (Bertrand \& Camos, 2015; Istomina, 1975), we aimed at dissociating their respective influence. A shopping stall was used as exogenous cue and walking played the role of an endogenous cue. The shopping stall provides an 
exogenous cue because it helps maintaining why children had to memorize the series of fruits and vegetables, and walking can provide an endogenous cue as the end of the walk coincides with recall time. By orthogonally manipulating both kinds of cues, we designed four experimental conditions: with or without goal cue combined with or without motor activity. In line with works on the role of goal cueing in executive control tasks and on the role of motor activities in cognitive tasks, both kinds of cue can be beneficial in maintaining the goal and thus improve memory performance. However, Fitamen et al. (2019) provided evidence for an absence of effect of exogenous cues in WM tasks, questioning whether exogenous and endogenous cues may have different role in WM. Finally, the association of the two types of cue could create the optimal game-like situation akin to the condition in which Istomina (1975) and Bertrand and Camos (2015) observed improvement in preschoolers' WM performance. Such improvements in WM were expected in preschoolers because these young children have been described as not using information-maintenance strategies. However, older children who are supposedly able to use maintenance strategies and therefore do not need external help to maintain the goal were also enrolled in the experiment. Indeed, for these older children, one may suspect that the introduction of a walk could impair WM performance because it is an attentiondemanding task (Hausdorff, Zemany, Peng, \& Goldberger, 1999; Lajoie, Teasdale, Bard, \& Fleury, 1993) that may interfere with the execution of information-maintenance strategies relying on attention (Barrouillet et al., 2009; Camos \& Barrouillet, 2011).

\section{Method}

\section{Participants}

Ninety-nine 5-year-olds $\left(M_{\text {age }}=5 ; 0, S D=0 ; 4,42\right.$ girls), 83 6-year-olds ( $M_{\text {age }}=5 ; 11$, $S D=0 ; 4,37$ girls $)$, and 70 7-year-olds $\left(M_{\text {age }}=6 ; 11, S D=0 ; 4,30\right.$ girls $)$ took part in the experience. The experiments took place at the children's school in a quiet room. The experiment was approved by the local ethic committee. Finally, we gathered from the parents or legal guardians a consent form, and children gave their consent orally before beginning the experiment. In each age group, children were randomly assigned to one of the goal cue conditions (i.e., presence vs. absence of the shopping stall) and to one order of presentation of the motor activity conditions (i.e., with walk then no-walk vs. no-walk then walk).

After testing, twenty-nine 5-year-old and ten 6-year-old non-native French speakers were excluded due to poor instruction comprehension and absence of knowledge for the animal, fruit, and vegetable names in French. Data from one 7-year-old child were excluded due to dyspraxia, which did not allow him to finish the animal run task. Thus, the final sample included 70 5-year-olds, 73 6-year-olds, and 69 7-year-olds (Table 1). Because children were discarded after testing, this led to similar, but not equal, group size.

\section{Material and procedure}

The design was adapted from Bertrand and Camos (2015). The experiment had a mixed design with two between-subject variables (age and goal cue) and one within-subject variable (motor activity). Children were randomly assigned to one of two conditions of goal cue (with vs. without). Moreover, each child had to perform the task with and without motor activity. The order of presentation of the two conditions of motor activity was counterbalanced. To assess the homogeneity of the two experimental groups in terms 
of WM capacity, every child performed an animal run task (Majerus, Poncelet, Greffe, \& Van der Linden, 2006). The administration of this latter task took place before and on a different day than the experimental conditions.

In the animal run task, children were told that animals were going to take part in a race and through headphones, they listened to the name of the animals in the order animals crossed the finish line. To recall the animal list, children had to reproduce the sequence in order by placing cards representing the animals they heard on a drawn podium with the same number of places than the number of animals in the race. The list lengths started from two animals per list, up to seven. Four trials of each list length were presented. Two recall scores were computed: the number of correctly reproduced lists and the number of animals recalled in correct position in the lists.

In the first session with the animal run task, we assessed the distance each child can walk at her own pace in $4 \mathrm{~s}$ in a training trial and three test trials. The average distance walked by each child on the test trials determined the walking distance in the experimental conditions (see below). The distance was adapted for each child, and it was on average $4.6 \mathrm{~m}(S D=1.3)$ at 5 years, $5.3 \mathrm{~m}(S D=1.2)$ at 6 years, and $5.5 \mathrm{~m}(S D=1.2)$ at 7 years.

The second session involving the WM task took place a few days later ( 9 days on average). The WM task was introduced as a game of 'grocery shopping'. Before starting the task, the experimenter verified that the child recognized each plastic items representing fruits and vegetables. The fruits and vegetables (banana, tomato, orange, lemon, carrot) were selected to have French bi-syllabic names with high frequency (Lété, SprengerCharolles, \& Colé, 2004), but also different shapes and colours to be easily distinguished. In each condition, children had to memorize a list of 1-5 fruits and vegetables. Four series were presented in each length, a given item appearing only once in each series. Two lists were created, one per condition of motor activity (with vs. without) for each child. A trial started when the experimenter took one fruit and put it in an opaque tube-shaped bag, the child paying attention to the scene. The items were successively introduced in the bag at a roughly regular rate of one every second, each item being named by the experimenter. When all the items of the series were in the experimenter's bag, the child had to reproduce the series by asking to another experimenter (playing the merchant) to place fruits and vegetables one by one in a transparent bag to allow the visualization of the series. For each recall condition, the child proceeded to the next length if she produced perfect recall (i.e., correct fruits in correct order) on at least one trial of a given length. Each child had to reproduce series of items in two different conditions (with vs. without walking). According to their group, a shopping stall was either or not present in the testing room.

For the condition without cue and without walk, the child stayed standing in front of the experimenter. In the condition with cue and without walk, the procedure was similar, except that, the shopping stall being beside the experimenter, the child was standing in front of the shopping stall. In the condition without cue and with walk, the child walked straight in front of her after the presentation of the items and the experimenter's signal to 'go ahead', while in the condition with cue and with walk, the child walked straight in the direction of the shopping stall. In all conditions, a signal heard $4 \mathrm{~s}$ after the presentation of the items prompted the recall of the items. In the conditions with walk, this signal also stopped the child's walk. At that moment, when there was no cue (no shopping stall), the child turned over to face the experimenter. In the condition with cue, the merchant appeared on the other side of the shopping stall in front of the child. 
A span score was calculated for each child in each condition. Each correctly recalled series (i.e., in which all the items were correctly placed in the order of presentation) counted as one-fourth, and the total number of fourths added (Barrouillet et al., 2009; Bertrand \& Camos, 2015; Smyth \& Scholey, 1992). Data supporting the findings are fully accessible online ${ }^{1}$.

\section{Results}

All Bayesian statistical analyses were performed under JASP (2020) (0.14). For each dependent variable, a Bayesian analysis of variance (ANOVA) was performed using the default settings. The $\mathrm{BF}_{10}$ of each model (e.g., main effects only, main effects + interaction effects) was obtained by comparing it to the null model. Our primary aim in choosing Bayesian analyses was to allow the testing of the null hypothesis in particular for the exogenous cue effect because Fitamen et al. (2019) reported evidence for the absence of effect in another type of WM tasks. For the endogenous cue (walk) effect, no specific priors can be used in our analyses, because the two available studies on this topic were not able to disentangle the effects of the two types of cues, as mentioned in the introduction. Hence, we preferred to keep the default settings to avoid introducing non-reliable values of priors. $\mathrm{A} \mathrm{BF}_{10}$ of 3 or more is considered substantial evidence for the model of interest; $\mathrm{a}$ $\mathrm{BF}_{10}$ below one third and conversely $\mathrm{a}_{\mathrm{BF}}$ greater than 3 are considered substantial evidence for the null model and values around 1 indicate no substantial evidence either way (Dienes, 2014; Jeffreys, 1961). Similarly, we favoured the best model when its probability to account for the data was three times greater than the second-best model; otherwise, both models were taken into consideration, and the examination of the $\mathrm{BF}_{\text {inclusion }}$ of the effects included in the models helped choosing the model to favour.

Bayesian analyses of variance (ANOVAs) were performed on the number of correctly reproduced lists and on the number of animals correctly recalled in correct position, which are the two scores for the animal run task with age groups, goal cue conditions, and orders of presentation of the motor activity conditions as fixed variables (Table 1). Please note that the two latter factors are related to our WM span tasks but defined different subgroups that should have similar performance on the animal run task to be compared in the main task. For the former score, unsurprisingly, the analysis revealed that the best model included only the age group effect, $\mathrm{BF}_{10}=5.88 \times 10^{6} \pm 0.01 \%$. However, the second-best model had only a probability 1.8 smaller than the best model. It included the effect of the order of presentation of motor activity in addition to the age group effect, $\mathrm{BF}_{10}=3.18 \times 10^{6} \pm 2.52 \%$. For the second score, results were rather similar, as the best model included the age effect and the order of presentation effect, $\mathrm{BF}_{10}=5.36 \times 10^{6} \pm 3.24 \%$, while the second-best model included only the age effect, $\mathrm{BF}_{10}=1.11 \times 10^{6} \pm 0.01 \%$, but the best model had to be preferred with a 4.8 greater probability than the second model. To summarize, in both analyses, the age and the order of presentation of the motor activity account for differences in the animal run scores.

We then examined the $\mathrm{BF}_{\text {inclusion }}$ for each factor in the models (i.e., a main effect or an interaction effect), its value for each factor indicates the likelihood of the data under models that included a given factor compared to all models stripped of the factor, in other words, computed across matched models. Confirming the models, the $\mathrm{BF}_{\text {inclusion }}$ for the

\footnotetext{
' Data supporting the findings can be found by following the present link https://mfr.osf.io/render?url=https\%3A\%2F\%2Fosf.io\% 2Frxeqp\%2Fdownload
} 


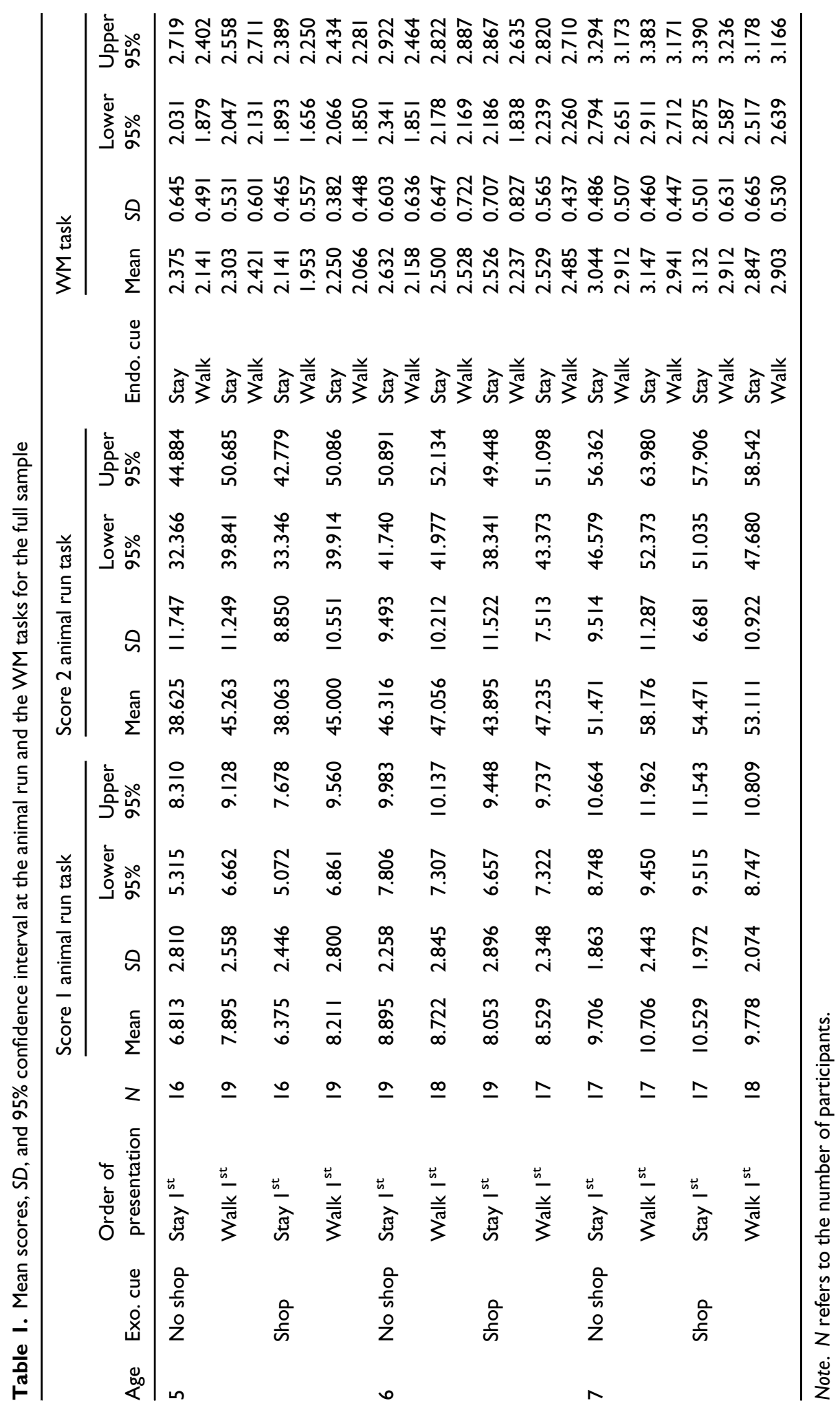


age effect was $6.71 \times 10^{6}$ and $2.33 \times 10^{8}$ for the first and second score of the animal run task, respectively. For the order of presentation, $\mathrm{BF}_{\text {inclusion }}$ was 0.55 and 4.82 , respectively. While the former value is anecdotical, the latter brought further support for the existence of a group difference according to the order of presentation conditions, as it also revealed in the models. For the effect of goal cue conditions, this analysis also confirmed the exclusion of this factor from the model. In both scores, the $\mathrm{BF}_{\text {exclusion }}{ }^{2}$ of the effect of the goal cue conditions was 5.70 and 5.71, respectively, and the $\mathrm{BF}_{\text {exclusion }}$ of the interaction between the age groups and the goal cue conditions was 9.19 and 11.47, respectively. The $\mathrm{BF}_{\text {exclusion }}$ of all the other interactions ranged between 2.49 and 11.47.

To summarize, we were rather confident that our following analyses performed on the recall score (span) of the task of interest would not be resulting from pre-experiment differences between the groups assigned to the two conditions of exogeneous cue (walk vs. no-walk). However, children assigned to the two orders of presentation of the motor activity unfortunately differed in their score in the animal run task. To take into account these differences in our analyses on spans, we ran two types of analyses to examine performance on our WM task, one on the full sample in which the two scores of the animal run task were introduced as covariables and another for which we created groups paired on their scores in the animal run task.

The first Bayesian ANOVA was performed on spans with the factors of interest (age, goal cue, and motor activity) and the two scores in the animal run task as covariables. The best model included the effect of age, the motor activity, and the two scores at the animal run task, $\mathrm{BF}_{10}=5.54 \times 10^{33} \pm 1.20 \%$. This model had a probability slightly greater than the second-best model, $\mathrm{BF}_{10}=4.59 \times 10^{33} \pm 1.06 \%$, which was similar to the best model but without the first score of the animal run task. The third model, which differed from the best model by the addition of the goal cue effect, provided a poorer account of the data, $\mathrm{BF}_{10}=1.80 \times 10^{33} \pm 1.74 \%$. We then examined the $\mathrm{BF}_{\text {inclusion }}$ for each factor in the models. Three factors were associated with a large $\mathrm{BF}_{\text {inclusion: }}$ the age effect, $\mathrm{BF}_{\text {incl. }}=6942.26$, the motor activity, $\mathrm{BF}_{\text {incl }}=859.91$, and the second score at the animal run task $\mathrm{BF}_{\text {incl. }}=42.48^{3}$. For the first score at the animal run task, the $\mathrm{BF}_{\text {incl }}$. was 1.29. Such BF value is considered as anecdotal at best (Jeffreys, 1998). All the other effects have

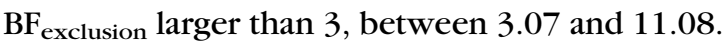

To run the second Bayesian ANOVA, we created paired groups. The previous analyses showed that larger differences between groups emerged for the second score at the animal run task that also appeared to account more for the data in the previous analysis than the first score at the animal run task. Hence, within each condition of goal cue and within each age group, we paired children from the two orders of presentation of the motor activity conditions on their second score at the animal run task (at $+/-$ one point). This pairing process led to discarding some children but to equal group sizes (Table 2). We checked that the pairing did result in the disappearance of differences on scores in the animal run task. A Bayesian ANOVA was performed on each score of the animal run task with age groups, goal cue condition, and order of presentation of the motor activity conditions as fixed variables. For both, the best model included only the age effect, $\mathrm{BF}_{10}=1.25 \times 10^{6} \pm 0.01 \%$ and $\mathrm{BF}_{10}=1.31 \times 10^{9} \pm 0.01 \%$, for the first and second scores, respectively. The second-best model, which included age and goal cue effects, had a probability 3.4 and 4.4 smaller than the best model, respectively. The examination of the

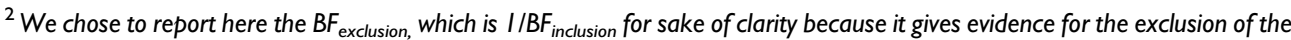
factor.

${ }^{3}$ Effect size for these effects was $\mathrm{n}_{\mathrm{p}}^{2}=.12,<.01$, and .05, respectively.
} 
$\mathrm{BF}_{\text {inclusion }}$ and $\mathrm{BF}_{\text {exclusion }}$ confirmed the best model, because the $\mathrm{BF}_{\text {inclusion }}$ for the age effect was $1.35 \times 10^{6}$ and $1.36 \times 10^{9}$ while the $\mathrm{BF}_{\text {exclusion }}$ for the goal cue effect was 3.45 and 4.38 , respectively. As expected, the $\mathrm{BF}_{\text {exclusion }}$ for the order of presentation was 5.39 and 5.14, respectively. The $\mathrm{BF}_{\text {exclusion }}$ for the interactions ranged from 3.25 to 7.84 , and 3.93 to 7.87 , respectively.

The second Bayesian ANOVA on spans of our WM task was run with age and goal cue as between-subject variables and motor activity as within-subject variable for children paired on the second score at the animal run task. The best model involved the effect of age and of motor activity, $\mathrm{BF}_{10}=8.87 \times 10^{9} \pm 6.0 \%$. This model had a probability 3.5 times greater than the second-best model to account for the data, $\mathrm{BF}_{10}=2.57 \times 10^{9} \pm 1.7 \%$. The second-best model included the three main effects of age, motor activity and goal cue. The examination of $\mathrm{BF}_{\text {inlcusion }}$ brought further support to the best model. The $\mathrm{BF}_{\text {inclusion }}$ for the age effect, $\mathrm{BF}_{\text {incl }}=5.91 \times 10^{7}$, and the motor activity, $\mathrm{BF}_{\text {incl }}=153.11^{4}$, were large, while the $\mathrm{BF}_{\text {exclusion }}$ exceeded 3 for the goal cue effect, $\mathrm{BF}_{\text {excl }}=3.45$. The $\mathrm{BF}_{\text {exclusion }}$ for the interactions ranged between 4.55 and 6.89, except for the age $\times$ goal cue interaction, for which the $\mathrm{BF}_{\text {exclusion }}$ was 1.18 . It could be noted that this interaction appeared on the third-best model, $\mathrm{BF}_{10}=2.24 \times 10^{9} \pm 5.4 \%$, which has a probability four times smaller than the best model.

Finally, a similar Bayesian ANOVA was performed with the addition of the order of presentation of the motor activity. The best model was the same as in the previous analysis with the effect of age and of motor activity, $\mathrm{BF}_{10}=8.45 \times 10^{9} \pm 2.1 \%$. This model had a probability 2.8 times greater than the second-best model to account for the data, $\mathrm{BF}_{10}=3.08 \times 10^{9} \pm 2.9 \%$. The second-best model included the effects of age, motor activity, order of presentation, and the interaction between the motor activity and its order of presentation. The third model, which involved the effect of age, motor activity, and goal cue had a probability 3.2 times smaller than the best model, $\mathrm{BF}_{10}=2.64 \times 10^{9} \pm 3.3 \%$. The examination of $\mathrm{BF}_{\text {inlcusion }}$ brought more support to the best model than to the second-best model, because only the $\mathrm{BF}_{\text {inclusion }}$ for the age effect, $\mathrm{BF}_{\text {incl }}=5.38 \times 10^{7}$, and the motor activity $\mathrm{BF}_{\text {incl }} .=150.43^{5}$, were large, while the goal cue, the order of presentation, and the interaction of the motor activity and its order of presentation were associated to $\mathrm{BF}_{\text {exclusion }}$ that support their exclusion from the model, $\mathrm{BF}_{\text {excl. }}=3.30,3.53$, and 4.48 , respectively.

To conclude, across the different analyses, our findings were rather congruent. Spans were unsurprisingly larger for older children, but decreased when children had to perform a motor activity. However, contrary to what was reported in previous studies, the introduction of a goal cue (here a shopping stall) had no effect on recall performance. Finally, these findings were not modulated by age.

\section{Discussion}

This experiment examined the impact of goal cue and motor activity on 5- to 7-year-old children's WM. Our aim was to disentangle the effect of exogenous and endogenous factors on recall performance. Our assumption was that children younger than 6-7 years of age would benefit from these two factors to effectively maintain the goal of the memory task and improve their recall performance. As exogenous cue, we introduced a shopping

\footnotetext{
${ }^{4}$ Effect size for these effects was $\mathrm{n}_{\mathrm{p}}^{2}=.07$ and $<.01$, respectively.

${ }^{5}$ Effect size for these effects remained $\mathrm{n}_{\mathrm{p}}{ }_{\mathrm{P}}=.07$ and $<.01$, respectively.
} 
stall in a WM span task akin to a shopping game. The endogenous cue was a motor (walking) activity, because walking is considered as a goal-oriented activity. Contrary to our hypotheses, we did not observe any improvement of WM performance in conditions with the shopping stall as potential goal cue in any of the age groups. Moreover, a detrimental effect of walking was observed in all age groups, contrary to our hypothesis that walking would only reduce recall performance in the older children because it would distract attention from attention-based maintenance activities. The results thus appeared at odds with those of Istomina (Istomina, 1975) and of Bertrand and Camos (Bertrand \& Camos, 2015), in which recall performance was improved in a game situation, which included both a goal cue and a motor activity. However, several elements in our experimental procedure differed from these previous studies.

First, in Bertrand and Camos' study (2015), children had to reconstruct the sequence of fruits and vegetables by retrieving in order from a box the previously memorized items. Our experience required a serial oral recall. This difference could account for the divergence in findings. Indeed, children involved in a reconstruction task may be less inclined to set up a maintenance strategy knowing that the items would be presented again during the recall phase. A reconstruction task can be performed by appealing only to the familiarity of the memory traces previously encoded in long-term memory (LTM) (see Yonelinas, 2002, for a review), without the active maintenance of information in WM, while serial recall tasks would rely on recollection through the retrieval of specific details of the memory items (Malmberg, 2008). Several dual-process models distinguish the two processes of familiarity and recollection (e.g., Jacoby, 1991; Mandler, 1980; Tulving, 1985; Yonelinas, 1994).

Second, contrary to our procedure in which items have to be maintained for a short 4second duration, Istomina reported delays of 60-90 s between the encoding and the recall phases. Recall after such long retention intervals indicated that information was probably retrieved from LTM in Istomina's procedure and not from WM. As a consequence, in both Bertrand and Camos (2015) and Istomina (1975), retrieval from LTM could (at least partly) underlie recall, while the serial oral recall after a short delay of retention used in our experiment requires an active maintenance in WM. Thus, the beneficial effects reported by Istomina (1975) and Bertrand and Camos (2015) may be specific to LTM processes. Further studies are needed to examine whether the impact of goal cue and motor activity on WM depends on the recall type. It may be suggested that the type of recall test would modulate the effects of cueing. According to the literature and the current study, cues should have a beneficial effect on preschoolers' performance in reconstruction tasks but not in serial recall tasks.

Our findings also questioned how motor activity impacts WM performance. By contrast to the literature that reported a beneficial effect of walking on WM performance in 9-year-olds (Schaefer, Lövdén, Wieckhorst, \& Lindenberger, 2010), we observed a detrimental effect when walking while maintaining information in 4- to 6-year-old children. Although Schaefer et al. (2010) reported a detrimental effect of walking when a lower walking pace was imposed to children, the beneficial effect appeared when children walked at their own pace. Indeed, children did walk at their own pace in the present experiment, as the distance to walk was defined for each child, based on a pretest, in order to keep the retention delay equivalent across participants. Nevertheless, walking did not result in any improvement in our experiment. Schaefer et al. (2010) explained their results by an activation of cognitive resources through walking that are then available for the WM task. On the contrary, we think that walking imposes a supplementary demand on attention, which may compete with the maintenance activities, like attentional 
refreshing (see Camos et al., 2018, for review). In fact, walking, even if it is relatively automatic and low-demanding in adults, requires the recruitment of some attentional resources to set the motor program up and carry it out correctly (Lajoie et al., 1993). However, maintaining information in WM also requires some attentional resources, particularly to implement an attentional refreshing. According to the time-based resourcesharing model (Barrouillet, Bernardin, \& Camos, 2004), these attentional resources will be shared between the maintenance of the information and any other task that requires attention, such as the walk in our study. As previously showed by Portrat, Camos, and Barrouillet (2010), the programming of a motor task can have detrimental effect on the concurrent maintenance of information in WM, an effect that is commensurate to the duration during which attention is needed for the motor programming. Nevertheless, this explanation would be plausible if young children were able to implement this attentional maintenance mechanism, which is exactly what we tried to trigger with the introduction of cues. Hence, the detrimental effect of walking can be conceived as a sign that young children actually implemented some maintenance strategies.

However, it remains possible to suggest that the detrimental effect of the motor activity may result from other differences between the walk and no-walk conditions. In the nowalk conditions, the auditory signal prompted the recall phase, while in the walk conditions, it has the dual function to stop the walk and prompt the recall. One can envision that such a dual function would increase cognitive load, resulting in the reduced memory performance observed in the walk conditions. However, we doubt that this was the case. In the walk conditions, when the shopping stall was present, children stopped walking when they arrived at the shop. Although the signal was played at about their time of arrival, being at the shop was the obvious end of their walking activity. Things are different when the shop was absent as in this condition the auditory signal has indeed this dual function to stop the walk and prompt recall. Hence, if the dual function of the signal induced a cognitive load that reduces memory performance, one should expect an interaction between the motor activity (walking conditions) and the goal cue (presence vs. absence of shop). However, our analyses did not provide any evidence in favour of this interaction. On the contrary, the BFs were for the exclusion of this interaction in the model, which led us abandon this alternative account to explain the detrimental effect of the motor activity.

Finally, although the walk has a detrimental effect on the memory performance, it should be noted that the effect size was rather modest in preschoolers with typical development. Although such an effect has strong implications for understanding memory functioning, it is rather fortunate that human motor activity does not impair strongly the WM maintenance of information, often required for directing action and solving the task at hand (see Baddeley, 2007, for the role of WM on action). Nevertheless, such a negative impact of a motor activity, even for a strongly automatized activity as walking, should not be neglected in educational settings, especially for children presenting learning difficulties (e.g., children with attention deficit hyperactivity disorder, ADHD). The concurrent performance of a motor activity often seen as a modest and negligible requirement can have detrimental consequences on cognitive performance. Recently, fidget spinners were sold as the miracle solution to the attention difficulties of ADHD children. However, their detrimental effect on attention, in line with the current findings, had been evidenced (Graziano, Garcia, \& Landis, 2020). Hence, avoiding or reducing as much as possible any kind of motor activities during a cognitive task requiring attentional resources in classroom as well as in reeducation should be recommended. 


\section{Conclusions}

To conclude, the present experiment provides evidence that exogenous cue does not help preschoolers to improve their WM performance. This contrasts with what is observed in executive control tasks, such as inhibition or task switching. As consequence, it can be put forward that the poor WM performance regularly reported in preschoolers is not related to any goal neglect that would hamper their maintenance strategies (see also Fitamen et al., 2019, for similar conclusion). Nevertheless, one can also suggest that this conclusion is restricted to WM task with serial recall and that performance in reconstruction or recognition tasks would benefit from the introduction of cues. Moreover, our findings also highlighted how a daily-practised motor activity such as walking can be demanding enough in young children to reduce their abilities to maintain information in short term. This result is in line with WM theories in which a common pool of domain-general resources has to be shared between controlled activities (e.g., Barrouillet \& Camos, 2015; Cowan, 2005; Engle, 2002). This also exemplifies how diverse are the tasks that can impact children's WM performance.

\section{Acknowledgements}

We thank the Direction de l'Instruction Publique of the Canton of Fribourg, the participating teaching teams, the children and their caretakers. We also thank D. Berard, F. Corminboeuf, A. Corthay, A. Despond, K. Hirsbrunner, M. Ngoy, C. Niçaise, D. dos Santos Sanches, M. Stritt, and C. Valnet for their help in collecting data.

\section{Funding}

This work was supported by the Swiss National Science Foundation and the Agence Nationale de la Recherche [Binational grant SNSF-100019L_156521 \& ANR-14-CE36-0011$01]$.

\section{Conflicts of interest}

The authors declare no conflict of interest.

\section{Author contributions}

Christophe Fitamen (Conceptualization; Data curation; Formal analysis; Investigation; Methodology; Project administration; Resources; Software; Supervision; Validation; Visualization; Writing - original draft; Writing - review \& editing) Agnès Blaye (Conceptualization; Funding acquisition; Methodology; Supervision; Writing - original draft; Writing - review \& editing) Valérie Camos (Conceptualization; Data curation; Formal analysis; Funding acquisition; Methodology; Project administration; Resources; Supervision; Validation; Visualization; Writing - original draft; Writing-review \& editing).

\section{Data availability statement}

Data supporting the findings can be found by following this link https://mfr.osf.io/render?url= https\%3A\%2F\%2Fosf.io\%2Frxeqp\%2Fdownload 


\section{References}

Allik, J. P., \& Siegel, A. W. (1976). The use of the cumulative rehearsal strategy: A developmental study. Journal of Experimental Child Psychology, 21(2), 316-327. https://doi.org/10.1016/ 0022-0965(76)90045-X

Baddeley, A. D. (2007). Working memory, thought, and action. Oxford, UK: Oxford University Press.

Barrouillet, P., Bernardin, S., \& Camos, V. (2004). Time constraints and resource sharing in adults' working memory spans. Journal of Experimental Psychology: General, 133(1), 83-100. https://doi.org/10.1037/0096-3445.133.1.83

Barrouillet, P., \& Camos, V. (2015). Working memory: Loss and reconstruction. Hove, UK: Psychology Press.

Barrouillet, P., Gavens, N., Vergauwe, E., Gaillard, V., \& Camos, V. (2009). Working memory span development: A time-based resource-sharing model account. Developmental Psychology, 45 (2), 477-490. https://doi.org/10.1037/a0014615

Bertrand, R., \& Camos, V. (2015). The role of attention in preschoolers' working memory. Cognitive Development, 33, 14-27. https://doi.org/10.1016/j.cogdev.2014.10.002

Blaye, A., \& Chevalier, N. (2011). The role of goal representation in preschoolers' flexibility and inhibition. Journal of Experimental Child Psychology, 108(3), 469-483. https://doi.org/10. 1016/j.jecp.2010.09.006

Camos, V., \& Barrouillet, P. (2011). Developmental change in working memory strategies: From passive maintenance to active refreshing. Developmental Psychology, 47(3), 898-904. https:// doi.org/10.1037/a0023193

Camos, V., \& Barrouillet, P. (2018). Working memory in development. Hove, UK: Routledge.

Camos, V., Johnson, M., Loaiza, V., Portrat, S., Souza, A., \& Vergauwe, E. (2018). What is attentional refreshing in working memory? Annals of the New York Academy of Sciences, 1424(1), 19-32. https://doi.org/10.1111/nyas.13616

Chevalier, N., \& Blaye, A. (2008). Cognitive flexibility in preschoolers: The role of representation activation and maintenance. Developmental Science, 11(3), 339-353. https://doi.org/10.1111/ j.1467-7687.2008.00679.x

Chevalier, N., \& Blaye, A. (2009). Setting goals to switch between tasks: Effect of cue transparency on children's cognitive flexibility. Developmental Psychology, 45(3), 782-797. https://doi.org/ $10.1037 / \mathrm{a} 0015409$

Cook, S. W., \& Goldin-Meadow, S. (2006). The role of gesture in learning: Do children use their hands to change their minds? Journal of Cognition and Development, 7(2), 211-232. https://doi.org/ $10.1207 / \mathrm{s} 15327647 \mathrm{jcd} 0702 \_4$

Cowan, N. (2005). Working memory capacity. New York, NY: Psychology Press.

Cowan, N. (2016). The many faces of working memory and short-term storage. Psychonomic Bulletin E Review, 24(4), 1158-1170. https://doi.org/10.3758/s13423-016-1191-6

Dienes, Z. (2014). Using Bayes to get the most out of non-significant results. Frontiers in Psychology, 5, 781. https://doi.org/10.3389/fpsyg.2014.00781

Engle, R. W. (2002). Working memory capacity as executive attention. Current Directions in Psychological Science, 11(1), 19-23. https://doi.org/10.1111/1467-8721.00160

Fitamen, C., Blaye, A., \& Camos, V. (2019). The role of goal cueing in kindergarteners' working memory. Journal of Experimental Child Psychology, 187, 104666. https://doi.org/10.1016/j. jecp.2019.104666

Flavell, J. H., Beach, D. H., \& Chinsky, J. M. (1966). Spontaneous verbal rehearsal in a memory task as a function of age. Child Development, 37(2), 283-299. https://doi.org/10.1111/j.1467-8624. 2007.01019.x

Gathercole, S. E., Lamont, E., \& Alloway, T. P. (2006). Working memory in the classroom. Working Memory and Education, 21(5), 219-240. https://doi.org/10.1016/B978-012554465-8/50010-7 
Goldin-Meadow, S., Nusbaum, H., Kelly, S. D., \& Wagner, S. (2001). Explaining math: Gesturing lightens the load. Psychological Science, 12(6), 516-522. https://doi.org/10.1111/1467-9280. 00395

Graziano, P. A., Garcia, A. M., \& Landis, T. D. (2020). To fidget or not to fidget, that is the question: A systematic classroom evaluation of fidget spinners among young children with ADHD. Journal of Attention Disorders, 24(1), 163-171. https://doi.org/10.1177/1087054718770009

Gruber, O., \& Goschke, T. (2004). Executive control emerging from dynamic interactions between brain systems mediating language, working memory and attentional processes. Acta Psychologica, 115(2-3), 105-121. https://doi.org/10.1016/j.actpsy.2003.12.003

Hausdorff, J. M., Zemany, L., Peng, C.-K., \& Goldberger, A. L. (1999). Maturation of gait dynamics: Stride-to-stride variability and its temporal organization in children. Journal of Applied Physiology, 86(3), 1040-1047. https://doi.org/10.1152/jappl.1999.86.3.1040

Hitch, G. J., \& Halliday, M. S. (1983). Working memory in children. Philosophical Transactions of the Royal Society of London. Series B, Biological Sciences, 302(1110), 325-340. http://www. jstor.org/stable/2395997

Istomina, Z. M. (1975). The development of voluntary memory in preschool-age children. Soviet Psychology, 13(4), 5-64. https://doi.org/10.2753/RPO1061-040513045

Jacoby, L. L. (1991). A process dissociation framework: Separating automatic from intentional uses of memory. Journal of Memory and Language, 30(5), 513-541. https://doi.org/10.1016/0749596X(91)90025-F

JASP, T. (2020). JASP (Version 0.14) [Computer software]. Retrieve from https://jasp-stats.org/.

Jeffreys, H. (1961). Small corrections in the theory of surface waves. Geophysical Journal of the Royal Astronomical Society, 6(1), 115-117. https://doi.org/10.1111/j.1365-246X.1961.tb 02965.x

Jeffreys, H. (1998). Theory of probability. Oxford, UK: Oxford University Press.

Lajoie, Y., Teasdale, N., Bard, C., \& Fleury, M. (1993). Attentional demands for static and dynamic equilibrium. Experimental Brain Research, 97(1), 139-144. https://doi.org/10.1007/ BF00228824

Lété, B., Sprenger-Charolles, L., \& Colé, P. (2004). MANULEX: A grade-level lexical database from French elementary school readers. Behavior Research Methods, Instruments, \& Computers, 36 (1), 156-166. https://doi.org/10.3758/BF03195560

Majerus, S., Poncelet, M., Greffe, C., \& Van der Linden, M. (2006). Relations between vocabulary development and verbal short-term memory: The relative importance of short-term memory for serial order and item information. Journal of Experimental Child Psychology, 93(2), 95-119. https://doi.org/10.1016/j.jecp.2005.07.005

Malmberg, K. J. (2008). Recognition memory: A review of the critical findings and an integrated theory for relating them. Cognitive Psychology, 57(4), 335-384. https://doi.org/10.1016/j.c ogpsych.2008.02.004

Mandler, G. (1980). Recognizing: The judgment of previous occurrence. Psychological Review, 87 (3), 252. https://doi.org/10.1037/0033-295X.87.3.252

Marcovitch, S., Boseovski, J. J., \& Knapp, R. J. (2007). Use it or lose it: Examining preschoolers' difficulty in maintaining and executing a goal: REPORT. Developmental Science, 10(5), 559564. https://doi.org/10.1111/j.1467-7687.2007.00611.x

Marcovitch, S., Boseovski, J. J., Knapp, R. J., \& Kane, M. J. (2010). Goal neglect and working memory capacity in 4- to 6-year-old children. Child Development, 81(6), 1687-1695. https://doi.org/10. 1111/j.1467-8624.2010.01503.x

Oftinger, A.-L., \& Camos, V. (2016). Maintenance mechanisms in children's verbal working memory. Journal of Educational and Developmental Psychology, 6(1), 16-28. https://doi.org/10.5539/ jedp.v6n1px

Oftinger, A.-L., \& Camos, V. (2017). Phonological similarity effect in children's working memory: Do maintenance mechanisms matter? Journal of Child Psychology, 1(1), 5-11. 
Oftinger, A.-L., \& Camos, V. (2018). Developmental improvement in strategies to maintain verbal information in working memory. International Journal of Behavioral Development, 42(2), 182-191. https://doi.org/10.1177/0165025416679741

Portrat, S., Camos, V., \& Barrouillet, P. (2010). Motor programming disrupt verbal maintenance. Revista Portuguesa de Pedagogia, 75-84. https://doi.org/10.14195/1647-8614_30anos_7.

Schaefer, S., Lövdén, M., Wieckhorst, B., \& Lindenberger, U. (2010). Cognitive performance is improved while walking: Differences in cognitive-sensorimotor couplings between children and young adults. European Journal of Developmental Psychology, 7(3), 371-389. https://doi. org/10.1080/17405620802535666

Smyth, M. M., \& Scholey, K. A. (1992). Determining spatial span: The role of movement time and articulation rate. Quarterly Journal of Experimental Psychology: Section A, 45(3), 479-501. https://doi.org/10.1080/02724989208250624

Swanson, H. L. (1999). What develops in working memory? A Life Span Perspective. Developmental Psychology, 35(4), 986. https://doi.org/10.1037/0012-1649.35.4.986

Tam, H., Jarrold, C., Baddeley, A. D., \& Sabatos-DeVito, M. (2010). The development of memory maintenance: Children's use of phonological rehearsal and attentional refreshment in working memory tasks. Journal of Experimental Child Psychology, 107(3), 306-324. https://doi.org/ 10.1016/j.jecp.2010.05.006

Towse, J. N., Lewis, C., \& Knowles, M. (2007). When knowledge is not enough: The phenomenon of goal neglect in preschool children. Journal of Experimental Child Psychology, 96(4), 320-332. https://doi.org/10.1016/j.jecp.2006.12.007

Tulving, E. (1985). Memory and consciousness. Canadian Psychology/Psychologie Canadienne, 26(1), 1. https://doi.org/10.1037/h0080017

Yanaoka, K., \& Saito, S. (2017). Developing control over the execution of scripts: The role of maintained hierarchical goal representations. Journal of Experimental Child Psychology, 163, 87-106. https://doi.org/10.1016/j.jecp.2017.06.008

Yonelinas, A. P. (1994). Receiver-operating characteristics in recognition memory: Evidence for a dual-process model.Journal of Experimental Psychology: Learning, Memory, \& Cognition, 20 (6), 1341. https://doi.org/10.1037/0278-7393.20.6.1341

Yonelinas, A. P. (2002). The nature of recollection and familiarity: A review of 30 years of research. Journal of Memory \& Language, 46(3), 441-517. https://doi.org/10.1006/jmla.2002.2864

Received I3 October 2020; revised version received I3 March 202 I 